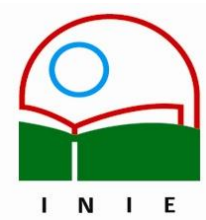

Actualidades Investigativas en Educación

Revista Electrónica publicada por el

Instituto de Investigación en Educación

Universidad de Costa Rica

ISSN 1409-4703

http://revista.inie.ucr.ac.cr

COSTA RICA

\title{
LIDERAZGO Y MOTIVACIÓN EN EL AMBIENTE EDUCATIVO UNIVERSITARIO
}

LEADERSHIP AND MOTIVATION IN THE UNIVERSITY EDUCATIONAL ENVIRONMENT

Volumen 9, Número 3

pp. 1-18

Este número se publicó el 15 de diciembre 2009

Yajaira Alvarado

Ana Teresa Prieto Sánchez

Daryeling Betancourt

La revista está indexada en los directorios:

LATINDEX, REDALYC, IRESIE, CLASE, DIALNET, DOAJ, E-REVIST@S,

La revista está incluida en los sitios:

REDIE, RINACE, OEI, MAESTROTECA, PREAL, HUASCARAN, CLASCO

Los contenidos de este artículo están bajo una licencia Creative Commons

(c) (i) () 


\title{
LIDERAZGO Y MOTIVACIÓN EN EL AMBIENTE EDUCATIVO UNIVERSITARIO
}

\author{
LEADERSHIP AND MOTIVATION IN THE UNIVERSITY EDUCATIONAL ENVIRONMENT
}

\author{
Yajaira Alvarado ${ }^{1}$ \\ Ana Teresa Prieto Sánchez \\ Daryeling Betancourt ${ }^{3}$
}

\begin{abstract}
Resumen: Las tareas del líder con respecto a la motivación son muchas y variadas. Entre otras cosas, un líder debe reconocer la necesidad de sus seguidores, ayudarlos a ver como pueden concretar esas necesidades y darles la confianza para que puedan alcanzar a remover constricciones o inhibiciones que han impedido la motivación. Esta inquietud ha sido la impulsora del presente artículo, que tiene como objetivo analizar el liderazgo y la motivación en el ambiente educativo universitario, concibiendo como líder al docente y como seguidores a sus estudiantes. Se trata de establecer las percepciones de los docentes en referencia al liderazgo que debe ejercerse en el ambiente educativo universitario. La fundamentación teórica se realizó sobre los aportes de Cooper y Sawf (2004), Robbins (2005), Goleman (2001), Bennis (2002), entre otros. El estudio es descriptivo, transversal, con un diseño de campo no experimental. El instrumento de recolección de datos fue el cuestionario y las entrevistas en profundidad. La población estuvo conformada por 72 docentes participantes del curso vacacional 2008 de la Universidad del Zulia, Venezuela, en el Núcleo Costa Oriental del Lago por ser una población finita se empleó el censo poblacional. Los resultados revelaron que existen mayores niveles de motivación cuando el liderazgo es de tendencia transformacional, donde la participación, el respeto y el compromiso son parte del trato que se brindan en la relación docente-estudiante.
\end{abstract}

Palabras Clave: LIDERAZGO, MOTIVACIÓN, EDUCACIÓN SUPERIOR, UNIVERSIDAD NACIONAL PÚBLICA.

\begin{abstract}
The leaders with motivation tasks are many and varied. Among other things, a leader must recognize the need for their followers, help you see how they can identify these needs and give them the confidence to bring up to remove constriction or inhibitions which prevented the of motivation. This concern was the instigator of this article, which aims to analyze the leadership and motivation in the University, educational environment developing as leader teacher and followers to their students. It is for teachers perceptions regarding leadership which must be exercised in the University educational environment. The theoretical foundation was made on the contributions of Cooper and Sawf (2004), Robbins (2005), Goleman (2001), Bennis (2002), among others. The study is descriptive, transversal, with a design not experimental field. The data collection instrument was the questionnaire and in-depth interviews. The population was composed 72 teaching participants holiday course 2008 the University of Zulia, Venezuela, in the core East Lake coast as a finite population employed population census. The results revealed that there are higher levels of motivation when leadership is transformational, trend where participation, respect and commitment are part of the treatment provided at the docente-estudiante relationship.
\end{abstract}

Key words: LEADERSHIP, MOTIVATION, SUPERIOR EDUCATION, PUBLIC NATIONAL UNIVERSITY

\footnotetext{
${ }^{1}$ Doctora en Ciencias Gerenciales. Profesora Asociada de la Universidad del Zulia en el Núcleo Costa Oriental del Lago, Venezuela.

Dirección electrónica: yaalv@hotmail.com

${ }^{2}$ Doctora en Ciencias Humanas. Profesora Titular de la Universidad del Zulia. Jefa Editora de la Revista Arbitrada Impacto Científico del Núcleo Costa Oriental del Lago de LUZ. Secretaria Docente de la Coordinación de Postgrado e Investigación. Investigadora acreditada en el Programa de Promoción del Investigador, Nivel II. Dirección electrónica: anaprieto@cantv.net anatere633@hotmail.com

${ }^{3}$ Cursante de la Maestría Gerencia de Operaciones de la Universidad del Zulia en el Núcleo Costa Oriental del Lago, Venezuela.

Dirección electrónica: daryelinb@hotmail.com
}

Artículo recibido: 4 de febrero, 2009

Aprobado: 30 de noviembre, 2009 


\section{Introducción}

El liderazgo siempre ha sido una cuestión importante dentro de la vida humana, nadie duda de la importancia de tener buenos líderes en el gobierno de un estado o en la administración o en la empresa. Es decir, los seres humanos, la sociedad en general y las organizaciones en particular necesitan líderes para progresar.

En la actualidad, el liderazgo es un punto crucial para que las organizaciones y empresas permanentemente sean más competitivas, lo que ha generado que las personas que las conforman sean eficientes y capaces de dar mucho de sí para el bienestar de la organización o empresa. Al hablar de organizaciones y personas es indispensable mencionar a los conductores, los líderes de hoy, aquellos que logran el éxito de sus organizaciones y que orientan a sus seguidores a conseguirlo.

El líder, como toda persona, posee muchos defectos y virtudes que debe conocer; esto implica conocerse a sí mismo, para luego entender a los demás, reflejar lo que quiere lograr, y qué busca alcanzar con los demás para conseguir el éxito. Este análisis buscará mejorar el desempeño como líder para ir desde lo particular hasta lo general, es decir, desde nuestro desempeño como líderes, sea para beneficio personal, hasta el desempeño en nuestra organización.

Ahora bien, el éxito de un líder depende, en gran medida, de la utilización efectiva de las estrategias motivacionales (Madrigal, 2005). En efecto, Berta Madrigal considera que liderazgo y motivación están estrechamente interrelacionados, lo que supone que todo líder debe imprimir energía a sus seguidores motivándolos al logro de objetivos visionarios. Debe poseer y demostrar sus capacidades para lograr motivar, porque de ello dependerá, fundamentalmente, su desarrollo y permanencia como tal.

Mc Farland y otros (2000) afirman que el mundo se mueve gracias al aporte de gente altamente motivada, gente que tiene creencias muy firmes u objetivos de urgente realización. Esa gente puede descarrilarse: conocemos los excesos nacidos del fanatismo, del odio, de la ambición y del deseo de poseer. Pero ninguna empresa se hace realidad sin hombres y mujeres fuertemente motivados. Cualquier asunto en el que las personas ofrecen su energía puede ser fuente de motivación. 
Las tareas del líder con respecto a la motivación son muchas y variadas. Entre otras cosas, un líder debe reconocer la necesidad de sus seguidores, ayudarlos a ver cómo pueden concretar esas necesidades y darles la confianza para que puedan alcanzar a remover constricciones o inhibiciones que han impedido el juego de la motivación.

Ante esta realidad, no escapan las organizaciones educativas, concibiendo como líder al docente y como seguidores a sus estudiantes, en este sentido, el docente deberá ejercer un liderazgo efectivo a través de la motivación, para administrar eficazmente su rol académico, con lo cual, sus estudiantes se conviertan en seguidores de sus conocimientos, altamente motivados, de manera que alcancen satisfacer sus deseos y necesidades.

Surge en este contexto la inquietud del presente artículo, el cual aborda una reflexión sobre el liderazgo y la motivación, con el objetivo de establecer las percepciones de los docentes en referencia al liderazgo que debe ejercerse en el ambiente educativo universitario. Para ello, se fundamentó teóricamente sobre la base de los aportes de Cooper y Sawf (2004), Robbins (2005), Goleman (2001), Bennis (2002), entre otros.

Para el logro del objetivo se estructuró la metodología como un estudio descriptivo, transversal, con un diseño de campo, no experimental. La población seleccionada estuvo conformada por 72 docentes participantes del curso vacacional 2008 de la Universidad del Zulia, Venezuela, en el Núcleo Costa Oriental del Lago. Se empleó el censo poblacional, y como instrumento de recolección de datos se utilizó el cuestionario y las entrevistas en profundidad.

Para profundizar más sobre lo anteriormente planteado, las próximas líneas buscan una sistematización precisa y concisa de algunos de los resultados más importantes que investigaciones precedentes han aportado con respecto a las teorías relacionadas con liderazgo y motivación en el ambiente educativo.

\section{Liderazgo}

El liderazgo es un proceso que consiste en influir en los seguidores para que alcancen metas, es decir, para que dirijan su conducta hacia determinados fines, de ahí su importancia Volumen 9, Número 3, Año 2009, ISSN 1409-4703 
dentro del proceso administrativo en cualquier organización, pues consiste en influir sobre las personas para que se esfuercen voluntaria y entusiastamente y logren las metas del grupo.

Sobre este particular, cabe destacar que no existe una única definición de liderazgo. Comprender esto es el primer paso para convertirse en un líder eficaz. Por ello, en este aparte se intentan analizar algunas definiciones y su papel en el ámbito educativo.

Para Lussier (2005) liderazgo es el proceso de influencia de líderes y seguidores para alcanzar los objetivos de la organización mediante el cambio. Es entonces necesario que el proceso de influencia se de recíprocamente entre líderes y seguidores, y no sólo del líder a su seguidor. Se trata, entonces de saber cómo dirigir y cómo desarrollar destrezas.

En el mismo orden de ideas, Chiavenato (2003) destaca el liderazgo como la influencia interpersonal ejercida en una situación, dirigida a alcanzar uno o diversos objetivos específicos. Siendo el liderazgo una cualidad propia de algunos gerentes, entonces, es necesario determinar aspectos propios de los mismos, tales como: capacidad para usar el poder con eficacia y, de un modo responsable, capacidad para comprender que los seres humanos tienen diferentes fuerzas de motivación en distintos momentos y en situaciones diferentes, capacidad para inspirar y capacidad para actuar en forma tal que desarrolle un ambiente que conduzca a responder a las motivaciones y fomentarlas.

Por otra parte, Elliott (2004) define el liderazgo como la responsabilidad que poseen algunos roles -aunque no todos- de influir en una o más personas (los seguidores) para que acepten voluntariamente los objetivos y finalidades propuestos por el líder, de modo tal que todos avancen en la dirección fijada por éste, infundiendo autoridad a las practicas de liderazgo propias de dicho rol.

De igual modo, Bouditch citado por Soto (2001) define liderazgo como el esfuerzo que se efectúa para influir en el comportamiento de los otros o para ordenar que se alcancen los objetivos organizacionales, individuales o personales. 
En este sentido, la función primaria de un líder debe consistir en producir cambios, para ello, debe estar en capacidad de generar un comportamiento pleno de energía que marque el rumbo y permita coordinar a quienes han de seguirlo. Marcar un rumbo identifica un camino idóneo a seguir, una sincronización eficaz hace que la gente avance por dicho camino y un esfuerzo motivacional que se vea coronado por el éxito garantiza que esas personas tengan la energía suficiente para superar los obstáculos que encuentren en su camino.

Para Robbins (2005) el liderazgo es esencial en los negocios, el gobierno e incontables grupos y organizaciones que conforman el modo en que vivimos, trabajamos y jugamos. Para este autor es la capacidad de influir en un grupo para la consecución de metas. Señala el autor que la fuente de esta influencia puede ser formal, como la posesión de un puesto gerencial en la organización, con grado de autoridad designada formalmente. Pero no todos los líderes son gerentes ni todos los gerentes líderes. El hecho de que las empresas provean a sus gerentes de ciertos derechos no garantiza que serán líderes eficaces. Asimismo, Stoner, Freeman y Gilbert (2003) consideran el liderazgo como el proceso de dirigir (ejercer el poder) las actividades laborales de los miembros de un grupo y de influir en ellas.

En este sentido la definición de liderazgo está ligada a la noción de poder. Blanchard define el poder como: "la capacidad de afectar (causar efecto en) el comportamiento de las organizaciones... tener poder es tener la capacidad de conseguir que determinadas cosas se hagan, de causar efecto sobre las acciones y decisiones que se toman" (Blanchard, 2000:5). De acuerdo con esta definición, el poder se puede ejercer tanto en las decisiones como en las acciones, siendo los líderes agentes de influencia social caracterizados por el ejercicio del poder en los equipos y departamentos que conforman las organizaciones.

Recientemente, y en la literatura sobre liderazgo, se ha hecho la distinción entre aquellos modelos centrados en un liderazgo transaccional y un liderazgo transformacional. Los modelos transaccionales asumen que los líderes deben ganar legitimidad para poder ejercer influencia. Entre los factores que pueden afectar esta legitimidad están: la conformidad de los líderes a las normas grupales, la competencia del líder en la tarea grupal y la fuente de autoridad del líder (Blanchard, 2000). 
Las teorías transaccionales de liderazgo se centran en cómo los líderes pueden motivar a sus seguidores creando intercambios justos y clarificando beneficios y responsabilidades mutuas; las teorías de liderazgo transformacional, por su parte, proponen que el líder debe estimular a sus iguales y seguidores para que consideren sus trabajos desde distintas perspectivas; hacerles conscientes de la misión o visión del equipo y de la organización; facilitar el desarrollo máximo de todo su potencial y motivarles para que, más allá de sus propios intereses, tengan en cuenta los intereses que benefician al grupo (Casado, 2000).

En ambas teorías, sin embargo, el énfasis está puesto en el rol del líder para el logro de los objetivos organizacionales. En las teoría de liderazgo transaccional la influencia del líder se centra sobre todo en el desarrollo óptimo de las tareas, mientras que en el liderazgo transformacional la influencia se orienta más bien hacia los valores que gobiernan el equipo y la organización, siendo el líder un agente de cambio.

A pesar de la importancia indiscutible que tienen los líderes en las organizaciones y de los múltiples enfoques teóricos y maneras de definir lo que se entiende por liderazgo, se estima que en las organizaciones actuales el liderazgo debe ser entendido en un contexto de equipos de trabajo en la organización. El análisis realizado, sobre la definición del liderazgo, conlleva a afirmar que forma parte de la influencia significativa que ejerce el líder en toda actividad con relación a los integrantes de un equipo, para que este funcione satisfactoriamente en el logro de los objetivos propuestos. Ante esta realidad no escapan las organizaciones de naturaleza educativa.

El liderazgo transformacional considera los valores y las percepciones de los seguidores como lo más importante, articulando los problemas conforme al sistema actual y distingue una visión nueva de la sociedad o de la organización. Esta visión está ligada a los seguidores y al líder y en congruencia con los valores que comparten entre ellos. De acuerdo con Casado (2000), el liderazgo transformacional es un ejercicio moral que sirve para incrementar el estándar de la conducta humana, el líder transformacional no solo es educado para cuidar los valores de los seguidores, sino para enseñarlos a ser líderes para que puedan convertirse en agentes morales. El líder trabaja en las etapas más altas de los valores morales de los seguidores y su visión rodea los ideales de éstos. Los líderes 
transformacionales aparecen en diferentes espacios sociales, por ejemplo, líder intelectual, reformador, revolucionario o heroico.

Los líderes transformacionales inspiran a sus seguidores a trascender sus intereses personales por el bien de la organización y son capaces de tener un efecto profundo y extraordinario sobre sus seguidores (Casado, 2000). Prestan atención a las inquietudes y necesidades de desarrollo de sus seguidores en lo individual; cambian la percepción de los problemas de sus subalternos al ayudarlos a ver sus antiguos problemas de una nueva forma, y son capaces de emocionar, despertar e inspirar a sus seguidores para que hagan un esfuerzo adicional que permita alcanzar las metas del equipo.

El liderazgo transformacional puede dar lugar a importantes cambios y resultados en la organización, ya que transforma al personal para que procure los objetivos de la organización en vez de sus propios intereses. Esta transformación de los seguidores, lo logra induciendo cambios en sus objetivos, valores, necesidades, creencias y aspiraciones. Los líderes transformacionales prestan atención individualizada, estímulo intelectual y poseen carisma.

En tal sentido se presenta a continuación un enfoque sobre el liderazgo docente enmarcado en un liderazgo transformacional, el cual consiste en crear desde lo que conocen los alumnos, de sus conocimientos previos, desarrollando estrategias que reconstruyan sus aprendizajes, y al cabo del tiempo, lo vuelvan a "desaprender-aprender".

\subsection{Liderazgo docente}

Es indudable que el docente siempre ha figurado como líder en su que hacer de formador, no obstante, su papel de liderazgo ha cambiado con el tiempo. Se requiere de docentes con compromisos; líderes transformacionales para seguir motivando la interacción social entre los equipos de trabajo en el aula. Este ejercicio de liderazgo transformacional consiste, por un lado, en inducir en otros el proceso continuo de mejoramiento y, por otro, estar en posición de influir para producir la innovación. Su esencia se centra en los siguientes aspectos: la toma de decisiones, la motivación y el empuje de los profesores en la innovación educativa. En éstos es trascendente la actitud de liderazgo transformacional. 
Según Ortiz (2007), el líder transformador es aquel que pone énfasis en los procesos; procura formar a las personas para cambiar la realidad; orienta una relación igualitaria entre él y sus alumnos donde todos aprenden de todos; crea las situaciones propicias para que se dé una educación permanente; desea hacer surgir una conciencia crítica; estimula la cooperación, la solidaridad y la creatividad y emplea como recursos didácticos los medios de comunicación masiva para analizarlos críticamente junto con sus alumnos.

Los procesos mencionados son los que facilitan la existencia de líderes transformadores, son los medios en donde se generan, de manera espontánea, las aptitudes, el carisma personal y el perfeccionamiento constante.

En este orden de ideas, el docente como líder transformacional promueve la inteligencia, racionalidad y emite solución cuidadosa de los problemas. Atiende y trata a cada persona de manera individual, capacita y aconseja. Es visionario, posee un proyecto de vida e involucra a sus seguidores hasta hacerlos identificarse con el proyecto.

Esta propuesta hace que el docente no se vea como el que sabe todo, se trata de contribuir con el alumno a que aprenda a construir su propio aprendizaje, a no sólo querer saber para aprobar un examen, sin analizar y sin discrepar de lo que el profesor propone como cierto.

Lo anteriormente planteado nos conduce a la necesidad de un nuevo papel de liderazgo, donde no se pase por alto que una relación de desigualdad puede atropellar el estilo democrático e integral que propone que en ese "enseñar-aprender" debe haber un actuación permanente que incluya a los actores del proceso, independientemente de los roles docenteestudiante. Los líderes transformacionales tienen varias formas de lograr todas estas tareas. La motivación es una de las más importantes. Para comprender esto, se requiere reflexionar sobre las diferentes accesiones de motivación.

\section{Motivación}

El ser humano es una criatura necesitada y raramente alcanza un estado de completa satisfacción, excepto en breves periodos de tiempo. Tan pronto se ha satisfecho un deseo, aparece otro en su lugar. Cuando éste se satisface, otro nuevo se sitúa en primer plano, y 
así sucesivamente. Es propio de los seres humanos desear algo, prácticamente siempre y a lo largo de toda su vida.

Para una mejor comprensión de lo planteado, en las próximas líneas se analizarán algunas definiciones sobre motivación, sin olvidar que el grado de ella varía entre las personas y dentro de cada persona según el momento.

Perreaut-Pierre (2000) la define como un conjunto de factores inconscientes que actúan sobre las conductas. Este autor parte del principio de que el comportamiento de cada persona viene determinado fundamentalmente por características innatas.

Para Varo (2003) la motivación es el proceso dirigido a despertar la acción, sostener la actividad en progreso y regular el patrón de actividad.

Por su parte Koontz y Weihrich (2004) la definen como un término general que se aplica a toda clase de impulsos, deseos, necesidades, anhelos y fuerzas similares.

Asimismo, Robbins (2004) la define como los procesos que dan cuenta de la intensidad, dirección y persistencia del esfuerzo de un individuo por conseguir una meta. La intensidad consiste en cuanto se esfuerza una persona.

Sin embargo, no es posible que una gran intensidad produzca buenos resultados de desempeño si el esfuerzo no se canaliza en una dirección que beneficie a la organización. Por tanto, se tiene que considerar la calidad del esfuerzo tanto como su intensidad. El esfuerzo que se debe buscar es el que se dirige hacia las metas.

Por último, la motivación tiene una vertiente de persistencia, que es la medida de cuanto tiempo sostiene una persona su esfuerzo. Los individuos motivados se aferran a una tarea lo suficiente para alcanzar la meta (Covey, 2002).

En cada una de estas definiciones se hace referencia a las necesidades o metas que el individuo se plantea satisfacer o conseguir; su estudio busca identificar las principales variables substantivas que motivan la conducta. Por otra parte, el proceso de motivación 
organiza la conducta en la búsqueda de obtener metas o de satisfacer las necesidades (Covey, 2002).

Ahora bien, en el campo educativo, la motivación consiste en fortalecer la voluntad de aprender del educando. Y para alcanzar este objetivo, el buen docente debe, ante todo, encontrar el motor principal capaz de impulsar la conducta del aprendiz.

En este sentido es preciso distinguir la conducta motivada por la propia acción, de la conducta que se emprende con el objeto de conseguir una recompensa. En el primer caso, se habla de motivación intrínseca y en el segundo de motivación extrínseca (Madrigal, 2005).

Por lo anterior, es vital relacionar la motivación extrínseca con las intrínsecas del docente, a fin de desarrollar un ambiente adecuado en el que se refleje una mayor productividad en el sistema educativo, contribuyendo al fortalecimiento del proceso enseñanza aprendizaje.

\section{Relación entre el liderazgo y la motivación}

La motivación es un fenómeno multicausal, originado por diversas fuentes, tales como los tipos de incentivos, reconocimientos, promociones, el trabajo por sí mismo, las necesidades de las personas, las metas y objetivos que se tengan, las condiciones de trabajo, los salarios, entre otros. Dentro de estas fuentes de motivación se encuentra el ejercicio del liderazgo.

Para Madrigal (2005) existe una relación directa entre el liderazgo que ejerce el líder y la motivación de sus seguidores, este vínculo entre ambos factores parece prevalecer sobre otro tipo de variables, entre estas se encuentran por ejemplo, las condiciones ambientales o sistemas de incentivos y su respectiva relación con la motivación.

Dentro de este contexto relacional, Prieto (2000) define la motivación como el elemento activador y director de una conducta hacia la consecución de una meta. Se distingue del aprendizaje en que este último explica la adquisición de conductas nuevas, pero no su activación ni su dirección. 
A un nivel general, la motivación extrínseca se puede explicar como la manera de inspirar a la gente, individualmente o en grupo, de forma que produzcan los mejores resultados posibles. En ese sentido, las organizaciones se han dado cuenta que deben alejarse de los métodos "comando y control" y acercarse a "aconsejar y acordar". Es decir, reconocen que premiar el buen trabajo es más efectivo que amenazar con castigar por un trabajo mal hecho.

Este enfoque es igualmente aplicable al ambiente educativo; si un estudiante siente que puede hacer lo que le satisface, sin presiones y castigos que lo rodeen, se podría afirmar que dicho estudiante cuenta con un ambiente propicio para el desarrollo de la motivación educativa.

Ante esto, comprendemos que, en el ámbito educativo, la motivación está asociada, de alguna manera, al liderazgo que se ejerce sobre esa persona, pues el estilo de liderazgo es uno de los factores que va a proveer el ambiente propicio para la elección personal sin que existan coerciones o castigos posteriores.

En este sentido, podría teorizarse estableciendo que el liderazgo asociado a niveles aceptables de motivación, sería aquel liderazgo realizado con una visión amplia del desarrollo humano, de manera que logre el avance social, integral y sostenible de cada persona. A la vez, esto trae como consecuencia que cada ser humano pueda llevar a cabo sus potencialidades en un marco de solidaridad, respeto y compromiso.

La persona que ejerce liderazgo no es la que suministra respuestas o da disposiciones para ser obedecidas al momento, sino aquella persona que guía los esfuerzos del grupo para definir sus propios problemas y encontrar sus propias soluciones.

Ahora bien, un docente puede lograr obediencia diciéndoles a sus estudiantes qué hacer, pero esto, por sí mismo, no modifica la comprensión y el compromiso de estos últimos; a largo plazo, y con un ambiente impositivo, traería como consecuencia: que se debiliten las capacidades del grupo para desenvolverse en un entorno complejo.

El proceso enseñar-aprender no consiste en tener a los estudiantes "robotizados". No se trata de procesar órdenes y dar soluciones inmediatas, todo lo contrario, se trata de que Volumen 9, Número 3, Año 2009, ISSN 1409-4703 
dicho proceso sea una actividad de carácter productivo, que vaya más allá de un "simplemente obedecer"; debe implicar cambios importantes para los seres humanos, tales como el desarrollo de los procesos de abstracción, representación psíquica de la realidad y su inserción en ella, elaboración de procesos de conciencia, entre otros.

En síntesis, si se ejerce un liderazgo que coarte las capacidades deliberativas y reflexivas de los estudiantes "robotizándolos", el proceso enseñanza-aprendizaje se vería truncada en toda su dimensión humana.

Aunado a lo anterior, el siguiente aparte tiene como propósito analizar algunas reflexiones en torno al papel que deben asumir los docentes universitarios en su rol de líderes educativos, bajo una perspectiva de la motivación en el contexto universitario. Además, presentamos los resultados más resaltantes obtenidos del trabajo de campo.

\section{Consideraciones metodológicas}

El tipo de investigación en el presente estudio, se calificó como descriptivo, transversal, con un diseño de campo no experimental. Ya que se analizaron las variables liderazgo y motivación en el contexto educativo universitario, tratando de concebir como líder al docente y como seguidores a sus estudiantes. Además los datos se recolectaron directamente en el lugar donde se desenvuelven las variables objeto de estudio.

Se utilizó un diseño no experimental pues se analizaron el liderazgo y la motivación realizando una sola medición de estudio, sin manipular deliberadamente las variables, sino ejecutar una descripción de sus comportamientos específicos.

La población objeto de esta investigación estuvo constituida por 72 docentes participantes del curso vacacional 2008 de la Universidad del Zulia, Venezuela, en el Núcleo Costa Oriental del Lago por ser una población finita se empleó el censo poblacional.

El instrumento de recolección de datos fue el cuestionario y las entrevistas en profundidad. Presentándose un formato que combinó preguntas cerradas y abiertas, además se incluyó 
preguntas de acción (para solicitar comportamientos relativos a actividades pasadas y futuras), mientras que las preguntas abiertas buscaron perfilar la opinión del entrevistado.

Las respuestas de los sujetos informantes fueron analizadas bajo el enfoque cualitativo y cuantitativo, así se cuantificó el porcentaje de docentes que manifestaron cierta tendencia en sus respuestas y se agruparon de manera cualitativa las respuestas mas significativas.

\section{Liderazgo y motivación en el contexto universitario. Análisis de resultados}

En la era de la postmodernidad, el saber no es absoluto, sino relativo. De ahí que se hable de ese proceso "aprender-desaprender-aprender". El docente no es aquel que tiene la verdad, sino el que sabe cómo construir en sus alumnos la forma de obtenerla. Lo que podríamos establecer como un proceso de intercambio de saberes entre educadoreducandos.

Por ello, dentro de las organizaciones es necesario un liderazgo efectivo, que sea considerado como una habilidad que se desarrolla en la medida en que el individuo cultiva la autoconfianza, el autocontrol y la perseverancia. De este modo, liderar implica empatía y capacidad de ilusionar a otros. En otras palabras, no es otra cosa que una gestión tanto de talento propio como ajeno, que conduce a una motivación entre los miembros de la organización.

Dentro de este contexto, esta investigación tuvo como finalidad analizar el liderazgo y la motivación entre docentes y estudiantes participantes del curso vacacional 2008 de la Universidad del Zulia en el Núcleo Costa Oriental del Lago, cuyos resultados se presentan a continuación:

El $75 \%$ de los docentes están conscientes de que debe de existir un liderazgo educativo que demande una modificación en la forma de actuar, precedido de una transformación de cómo piensa y siente en el salón de clase el estudiante, por lo cual es necesario un enfoque mental más flexible y dispuesto al cambio permanente del entorno. 
En este sentido, y dentro de la relación que existe entre el docente y sus estudiantes, el liderazgo es indispensable, pues forma estructuralmente un equipo y en todo momento, para tener el éxito deseado, ese equipo debe entender que cada uno forma parte de ello y el aporte y la realización de las actividades asignadas son de gran significancia para la obtención del resultado final deseado.

Un $100 \%$ de los docentes encuestados estuvieron de acuerdo con que el líder educativo debe ser visionario, colaborador, facilitador, con capacidad de resolver problemas y lograr consensos bajo los reglamentos y normativas de la institución educativa. Esto es lo que se conoce como liderazgo transformacional.

Este nuevo líder educativo debe reconocer que la comunidad universitaria establece demandas múltiples y a menudo divergente en la cultura organizacional, por lo que debe actuar fomentado por la filosofía del "nosotros" y no en la del "yo". Entonces, cuando se identifica con la misión institucional en sus distintas manifestaciones, puede ofrecer alternativas metodológicas viables para promover aprendizajes de actitudes y valores, y asume que con su ejemplo son tangibles tanto la visión como los valores institucionales.

De igual manera, el 100\% de los docentes opinaron que el líder educativo debe considerar las potencialidades y limitaciones de sus alumnos, de forma tal que pueda impulsar el talento de estos según sus manifestaciones personales.

Al respecto, Santiago (2004) define el liderazgo transformacional como una relación de influencia de trabajo guiada por una alta motivación de ambas partes, líder y seguidores. EI autor considera que en esta relación el colaborador interactúa con el líder por motivación extrínseca e intrínseca. El docente entrega lo mejor de sí mismo para conseguir lo mejor de los demás.

Caso contrario, y en un $90 \%$, el docente considera necesario infundir en sus estudiantes la curiosidad y el espíritu crítico, abordar el conocimiento desde la pregunta y el razonamiento, interactuar con sus compañeros y con todos los que hacen vida dentro de la comunidad universitaria, transmitirles la convicción absoluta del poder transformador del individuo -el poder de UNO- para crear y recrear su propia realidad, con una conciencia de Volumen 9, Número 3, Año 2009, ISSN 1409-4703 
responsabilidad social en este mundo globalizado en donde la solidaridad es el único puente que enlaza a los individuos.

En síntesis, se evidenció que el estilo de liderazgo fue considerado por los docentes como un factor determinante para generar motivación en los estudiantes. Ello coincide con lo planteado por Maslow en su obra: Una teoría sobre la motivación humana (en inglés, A Theory of Human Motivation) de 1943, que posteriormente amplió, quien señala que es muy importante sentirse reconocido en la organización donde se labora.

En relación a la variable motivación se pudo constatar que los docentes la consideran: generadora de expectativas para la excelencia, y como fuente inspiradora para ser un equipo de trabajo. Este factor es descriptivo del liderazgo transformacional de altas expectativas a los seguidores, inspirándoles, a través de la motivación, a estar comprometidos y ser parte de una visión compartida en la comunidad universitaria.

En la práctica, los líderes usan símbolos e incentivos emocionales para concentrar los esfuerzos de los miembros en lograr más de lo que harían en su propio interés personal. El espíritu de equipo es elevado por este tipo de liderazgo.

También, los docentes consideran importante que debe existir, en cada una de los miembros del proceso enseñanza - aprendizaje, la capacidad de motivarse a sí mismo. En ese sentido, Salovey (2000) expone que el automotivarse hace a las personas más alegres, productivas y eficaces en todas las tareas o actividades que emprenden. Realizar acciones para alcanzar un determinado objetivo genera la energía suficiente para superar los obstáculos y contratiempos que puedan surgir.

Además, un $100 \%$ de la población cree que cuando la motivación es suficiente, las probabilidades de lograr con éxito lo que se pretende aumentan considerablemente. En este sentido aplicar estrategias sobre dirección y desarrollo del personal son los factores más importantes que permitirán contribuir al logro de los objetivos planteados y al desarrollo personal de los individuos. 
Finalmente, manifiestan que se deben crear hábitos institucionales en los que día a día se valore el esfuerzo de los actores del proceso enseñanza-aprendizaje y que, sobre todo, se humanice el trato en la relación docente-estudiante. A modo de ejemplo, la clásica "palmadita en el hombro" es un detalle que anima al miembro del grupo y más si quien la proporciona tiene una jerarquía importante en el proceso. Consideran que mientras más confianza y reconocimiento se les proporcione, más se involucrarán y se sentirán comprometidos a responder, eficientemente, las tareas encomendadas.

\section{Consideraciones finales}

El análisis teórico presentado permite anotar las siguientes conclusiones:

Para lograr que el proceso de enseñanza aprendizaje se de con éxito es imprescindible que la motivación de las personas involucradas en dicho proceso esté determinada por el valor que asignen al resultado de sus esfuerzos, multiplicado por la confianza que tienen de que tales esfuerzos ayudarán a alcanzar las metas trazadas. En otras palabras, la motivación es un producto del valor anticipado que una persona le asigna a una meta y las posibilidades que ve de lograrlas

Con características de líderes transformacionales que acentúan el mejoramiento continuo, comprometiéndose con la optimización del proceso y del beneficio que éste supone para sus alumnos, los docentes alientan el cambio como necesidad sentida, porque impulsan la motivación en otros y promueven la creación de oportunidades de liderazgo para el empuje de las demás personas de la comunidad universitaria.

El docente debe guiar para que los demás tomen conciencia de que su labor, junto a otros agentes tradicionales, es causa y efecto de las decisiones y actuaciones de quienes forman parte de esa comunidad y que la particular contribución de cada uno de los profesores contribuirá al desarrollo de los estudiantes.

Todo docente debe ser un líder, ya que en el ejercicio de su función tiene que actuar sin demora, cuando ha tomado conciencia de la necesidad de cambiar la realidad en que se desarrolla.

Volumen 9, Número 3, Año 2009, ISSN 1409-4703 
Educar es un acto humano honorífico que no acepta demoras y mucho menos evasiones. Esta característica de agente de cambio se reafirma cuando la comunidad le atribuye al docente la calidad de creíble, pues él sabe qué quiere lograr de sus alumnos y tiene los conocimientos, las destrezas y la disposición para hacerlo y, además, asume que su rol educativo se ejercita en torno a un sistema coherente de valores y principios, los de la institución educativa donde se desenvuelve.

El docente debe tener la capacidad de crear y sostener una comunidad de valores compartidos donde se unifiquen voluntades, motivaciones, estrategias y responsabilidades declaradas para lograr consistencias y coherencias en las metas trazadas.

Es necesario que el docente sea un líder transformacional que se comprometa con su vocación y sus convicciones; que propicien el desarrollo personal, ético y profesional de todos los miembros de la comunidad universitaria, además, debe poseer una visión del futuro, para constituirse en asesor permanente de sus educandos.

El líder educativo insiste en los procesos aprender-desaprender-aprender y entiende el milagro, misterio y necesidad de un desarrollo para la cooperación y la solidaridad. Liderazgo implica un compromiso moral y ético que el docente transmite con su actitud personal; significa grandeza, cargada de todos los valores que como misión deben transmitirse a las nuevas generaciones.

\section{Referencias}

Bennis, Warren. (2002). Cómo llegar a ser líder. Colombia: Editorial Norma

Blanchard, Ken. (2000) El corazón de un líder. El arte de influir. México: McGraw Hill

Casado, José. (2000) EI Directivo del Siglo XXI. España: Ediciones Gestión 2000

Chiavenato, Idalberto. (2003). Introducción a la Teoría General de la Administración. México: Mc. Graw Hill

Cooper, Robert y Sawaf, Ayman. (2004) La inteligencia Emocional aplicada al liderazgo y a las organizaciones. Il edición. Colombia: Grupo editorial Norma 
Elliott Jaques. (2004). La organización requerida: Un sistema integrado para crear organizaciones eficaces y aplicar el liderazgo gerencial en el siglo XXI. Buenos Aires: Publicado por Ediciones Granica S.A.

Goleman, Daniel. (2001). La inteligencia emocional. España: Editorial Vergara

Koontz, Harold y O’Donnel, Cyril. (2004). Administración una perspectiva global. McGraw Hill, 10ª Edición. México

Kotter John. (2000). La verdadera labor de un líder. Colombia: Norma

Lussier Robert. (2005). Liderazgo: Teoría, aplicación, desarrollo de habilidades. México: Tomson Learning

Madrigal, Berta. (2005). Liderazgo. Enseñanza y aprendizaje. México: McGraw Hill Interamericana

McFarland, Lynne, Senn, Larry y Childress, Jhon. (2000). Liderazgo para el siglo XXI. Diálogos con 100 líderes destacados. Colombia: McGraw Hill Interamericana

Covey, Stephen. (2002). Los tres papeles que desempeña el líder en el nuevo paradigma. Colombia: Ediciones Deusto

Perreaut-Pierre, Edith. (2000) Sofrología y éxito deportivo: Guía práctica para los deportistas y para los que no lo son. México: Thompson

Prieto, José. (2000). Introducción a la psicología: Teoría y ejercicios. España: Editorial Ramón Areces

Santiago, Ginés. (2004). Ética y liderazgo empresarial: una complementariedad necesaria. Recuperado el 5 de octubre de 2008, de http://www.gobernabilidad.cl/modules.php?name=News\&file=arti...76k

Soto, Eduardo. (2001). Comportamiento organizacional: Impacto de las emociones México: Tomson Learning

Stephen, Robbins. (2004) Comportamiento organizacional: Conceptos, controversias y aplicaciones. Edition: 10 Publicado por Pearson Educación

Varo, Jaime. (2003). Gestión estratégica de la calidad en los servicios sanitarios: Un modelo de gestión hospitalaria. Edition: illustrated Publicado por Ediciones Díaz de Santos 